\title{
How to Avoid Giant Retinal Tear Slippage: New Surgical Approach
}

\author{
Mohamed Zidi ${ }^{1,2}$ \\ ${ }^{1}$ Nour Ophthalmology Clinic, Casablanca, Morocco \\ ${ }^{2}$ Faculty of Health Sciences, International University of Casablanca, Casablanca, Morocco
}

Email address:

zidiretine@hotmail.com

\section{To cite this article:}

Mohamed Zidi. How to Avoid Giant Retinal Tear Slippage: New Surgical Approach. International Journal of Ophthalmology \& Visual Science. Vol. 6, No. 2, 2021, pp. 122-127. doi: 10.11648/j.ijovs.20210602.20

Received: April 30, 2021; Accepted: May 31, 2021; Published: June 16, 2021

\begin{abstract}
Introduction: The retinal detachment (RD) by giant tear is a rare but serious clinical form. Apart from its therapeutic difficulty, during the fluid-air exchange, it poses a problem of retinal slippage which may be responsible for therapeutic failure or at best for retinal folds with serious anatomical and functional consequences. The purpose of this study is to shed light on the keys to the success of this technique. Material and methods: Retrospective study over 5 years from January 2014-December 2019 concerning 56 cases of retinal detachment by giant tear. All the patients were operated on by vitrectomy and laser endophotocoagulation of the tear edge and on $360^{\circ}$. The technique involved a first transient exchange of PFCL-air followed by a second exchange of silicone-air or gas-air depending on the case. Results: Intraoperatively, no cases of slipping, even in the cases of gas tamponade, have been observed. A primary retinal re-application of the RD was noticed in $87.5 \%$ of cases. The rate of retinal detachment recurrence was 12.5\% (all had an advanced vitreoretinal proliferation). After recurrence surgery, the final reapplication rate was $100 \%$. Conclusion: Mastering the PFCL-Air exchange during vitrectomy retinal detachments by giant tearing according to the technique described helps to prevent the slippage of the retina. The gas tamponade can be an alternative to silicone tamponade in some cases of giant tears selected with lower horns above the $4-8$ meridians.
\end{abstract}

Keywords: Retinal Detachment, Giant Tear, Slippage of the Retina, Fluid-Air Exchange

\section{Introduction}

Retinal detachment (RD) by giant retinal tear (GRT) is a rare but serious clinical form (represent $0.5-1.5 \%$ of the total number of rhegmatogenous detachments). A giant retinal tear is defined as a tear greater than or equal to $90^{\circ}$, sometimes it can reach $180^{\circ}$ or even $360^{\circ}$ with retinal inversion. Giant retinal tears occur most often among male myopic, with or without trauma (contusive or surgical), often associated with vitreoretinal degeneration [1]. Physiopathology is linked to an abnormality of the back of the vitreous base [2]. The surgical therapeutic difficulty of the retinal detachment by giant tear is mainly related to the retina reapplication, especially in case of advanced vitreoretinal proliferation (VRP).

Per operative difficulties and complications (retinal reinversion, retinal slippage, radial folds, tamponade passage under the retina or choroidal detachment) mainly occur at the time of the exchanges. Hence the importance of a good understanding of the mechanisms of the exchange and its mastering (especially in the case of fluid-air exchange) in order to maintain a high level of perfectly reapplied retina at the end of the surgery [3].

\section{Objectives}

The aim of our work is to share our experience in the field of surgery of Retinal detachment by giant tear and to describe the phenomenon of retinal slippage during an exchange airfluid according to a theory we have used based on a simulation with mathematical calculation in order to specify the correlation between the amount of subretinal liquid formed and the slipping distance or retinal shortening in relation to an initial reference point on the edge of the GRT under PFCL. Finally to discuss exchange air-fluid techniques and types of adequate tamponade in order to prevent complications linked to exchange, particularly in the case of 
tamponade by gas.

\section{Materials and Methods}

We conducted a descriptive retrospective study on 56 cases of patients operated for retinal detachment by giant tear. We noticed epidemiological data, the type of detachment (VRP, extent and location of the giant tear), the surgical technique, the type of tamponade and finally the anatomical and functional results. The minimum follow-up was 6 months.

\section{Results}

The average age of the patients was 40 with extremes of age ranging from 13 to 75 with a clear male predominance ( $84 \%$ of the population of the patients were male). Thirty-six percent of patients were very short-sighted (refraction $>-6$ diopters or axial length $>26 \mathrm{~mm}$ ). The initial acuity was less than $1 / 10$ in $85.7 \%$ of cases. Two post traumatic cases of retinal detachment by giant tear have been recorded. The final visual acuity was located between $1 / 20$ and $8 / 10$. The location of the giant tear was superior and temporal respectively in $19.64 \%$ and $41.07 \%$ of cases. The horn lower than the GRT was greater than the meridians of $4 \mathrm{~h}-8 \mathrm{~h}$ in only $11 \%$ of cases (i.e. 6 cases). VRP stage $C$ was noted in $75 \%$ of cases and only $25 \%$ of retinal detachments were at stage $\mathrm{A}$ or $\mathrm{B}$ of the VRP. A combined surgery was necessary in 2 given cases with the presence of an associated cataract. We operated on all patients using the vitrectomy technique without stitches, under local anesthesia (sub tenonian) including the 13 years old child. After central and peripheral vitrectomy and after the reapplication of the retina under PFCL, the first temporary exchange that has been used is PFCL-air exchange in $100 \%$ of the cases. The final exchange by silicone oil $5000 \mathrm{cSt}$ was used in $89 \%$ of cases. Nonexpansive gas tamponade has been carried out in 6 patients, including 4 cases of tamponade with $\mathrm{C} 2 \mathrm{~F} 6$ gas (at 16\%) and 2 cases of gas tamponade $\mathrm{C} 3 \mathrm{~F} 8$ (at $12 \%$ ).

Intraoperatively, no technical complications were observed. No cases of retinal slipping were noted with our exchange technique. Post-operatively, the rate of the primary retinal reapplication, The RD rate, was $87.5 \%$. No retinal folds were observed. The recurrence rate of retinal detachment was $12.5 \%$ (7 patients). It should be noted that all recurrences had an initial VRP stage C and the VRP was in lower location in all cases. Six had a silicone tamponade and 1 case had gas tamponade. After the resumption of these recurrence surgeries by vitrectomy, dissections of PVR, relaxation retinectomy depending on the case and silicone oil tamponade; the final replication rate goes back to 100 percent.

\section{Discussion}

Retinal detachment by giant tear is a serious form of retinal detachment. The prognosis has greatly improved on the anatomical and functional plan since the 1980s with the development of vitrectomy techniques and especially by the use of PFCL [4]. The indication of choice is the vitrectomy with the use of PFCL. However, there is still a controversy concerning the association of a vitrectomy with a localized external indentation or even surgical cerclage especially for tears in the lower area [5]. In our study, no case has been dealt with by an associated external indentation. Some authors use as retinopexy of the edges of the GRT a photocoagulation endolaser with cryo-application of GRT horns $[6,7]$. In our practice, retinopexy is performed only in the cases with argon laser $532 \mathrm{~nm}$ including for horns. A laser strapping $360^{\circ}$ has been systematically carried out for all patients in this study. The type of tamponade conditions and the type of exchanges are: PFCL-silicone which is widely used by many authors while the PFCL-air and then air-silicone or gas exchange is less produced in the published series $[8,9]$.

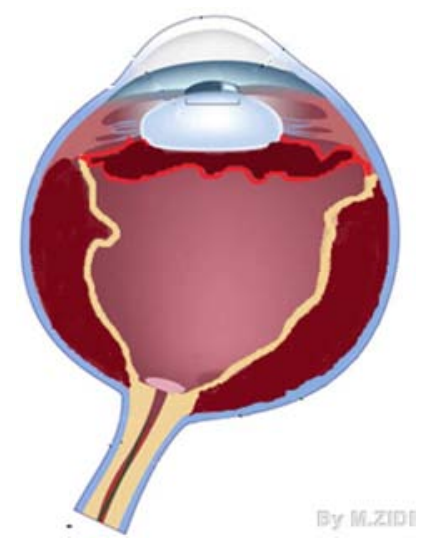

Figure 1. Illustration of a RD by GRT.

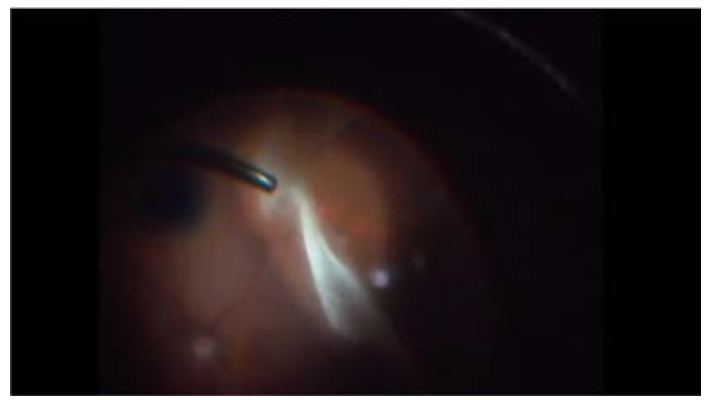

Figure 2. Patient M: Total retinal detachment by RGT: Vitrectomy.

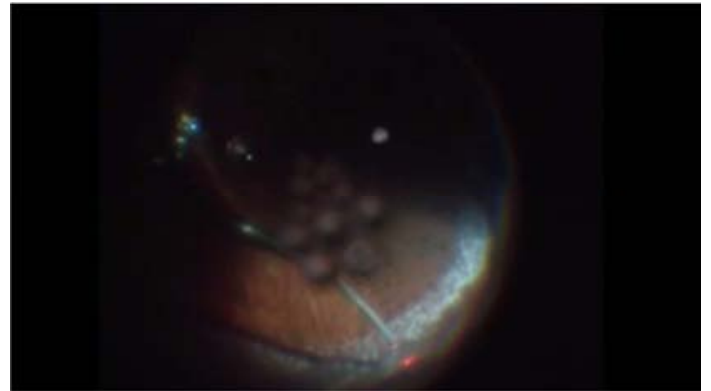

Figure 3. Patient M: Total retinal detachment by RGT: Edge endolaser under air and gas tamponade $\mathrm{C} 2 \mathrm{~F} 6$.

In the case of RD by GRT (Figure 1), the central and peripheral vitrectomy allows to suppress the pulls exerted on 
the horns of the GRT. Posterior vitreous detachment (PVD) is almost always present in this type of RD. The posterior edge of the detached retina is often free of any vitreous attachment except at the level of the 2 GRT horns. Diagnostic delay is a source of VRP formation which is the main cause of recurrence of RD by GRT. Dissection of this VRP is a critical time in the surgery to allow the retina to regain its suppleness and to reapply itself without folds or shrinkage. This dissection can be continued under retinal space and sometimes supplemented by work of horns loosening or even by a retinectomy relaxation. The injection of PFCL reapplies the posterior retina up to the posterior edge of the GRT and the horns [10]. The resection of the anterior retinal flaps is necessary to prevent VRP and to avoid the occurrence of neo-vascular glaucoma (NVG) postoperatively by retinal ischemia of the anterior flap of the GRT [11]. This resection will also avoid post-operative hypotonia operation by rolling up the anterior edge of the giant tear on the postoperative ciliary body. Retinopexy is obtained by endophotocoagulation with argon laser on the back edge of the tear and on $360^{\circ}$ with return to ora serrata [6] (Figure 2, 3 ). These steps are a prerequisite for the success of the exchanges in particular and of surgery in general.

The replacement of the $20 \mathrm{G}$ vitrectomy with sutures by the vitrectomy $23 / 25 / 27 \mathrm{G}$ without sutures [10] at the same time increased the comfort for the patient and the surgeon, but it has further complicated the exchanges due to the lack of a reinforced terminal as for the one used earlier for the $20 \mathrm{G}$, which limited for most of the surgeons the use of silicone oil to low viscosity silicones $(1000 \mathrm{cSt})$ instead of Silicone 5000 cSt. The latter must be the selected tamponade of RD by GRT (high tolerance low percentage of emulsion unlike 1000 cSt silicone) [12]. To have a successful exchange in the RD by GRT, both properties: Reapplication strength and tamponade power are set to contribution. The principle is to obtain a unique bubble that chases away effectively the subretinal liquid through the tear (by the force of the reapplication) and the obstruction of the tear (thanks to the tamponade power) [13] (Figure 4). The principle of PFCL Silicone exchange is to go from an internal tamponade of intermediate re-application force to an even weaker one. There is therefore less risk of the retina slipping. These two products have a high surface tension and therefore remain under the shape of a single bubble that does not pass under the retina. During the entire exchange process, the retina is in contact with the tamponade products. Visibility conditions are much better to remove a residual bubble of PFCL which pushes the majority of surgeons to use the silicone oil which will be injected by a direct exchange with silicone at the top and PFCL at the bottom [14]. The infusion terminal is then connected to the silicone pump with an infusion pressure at 1.5 bar, the infusion pressure injects Silicone oil with passive suction of PFCL [15]: this represents a time-consuming inconvenience with the risk of losing a lot of time in case of vitrectomy without suture (no reinforced terminal) and especially if we want to use high density silicone oil.

In our practice, and to overcome these difficulties; we have carried out a direct PFCL-Air exchange to have the choice of gas tamponade or silicone $5000 \mathrm{cSt}$. The subretinal liquid will be evacuated through the tear by the PFCL. For the posterior retina, the fluid under the retina is pushed from the back to the front by the PFCL bubble whereas after injection of the air that will first be in contact with the PFCL of the anterior retina, the liquid will be pushed from front to back (Figure 5). The main problem in this exchange is related to the persistence of sub-retinal liquid that can be trapped between the air at the top and the PFCL at the bottom. Any rush to suck up the PFCL before drying the serum well in front of the PFCL may cause a passage of the latter into the sub-retinal space (Figures 6,7). The air infusion is regulated at a pressure of about $30-35 \mathrm{mmHg}$. The suction by the head of the vitrectomy probe is then positioned just in front of the posterior edge of the giant tear to aspirate all the serum located between the insufflate air and the PFCL, avoiding any passage of the serum under the retina. The trick is to dry the liquid well before aspirating the PFCL, the exchange at this level is equivalent to an exchange during a macular hole surgery but regarding the edge of the giant tear. A passive suction is more than sufficient.

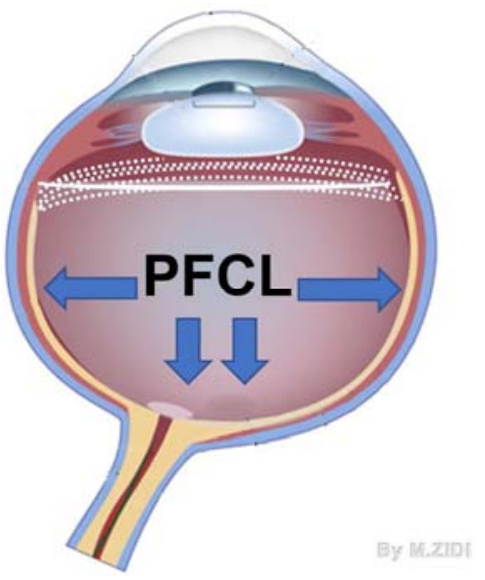

Figure 4. Reapplication of the detached retina by PFCL whose strength is practiced from the bottom to the side.

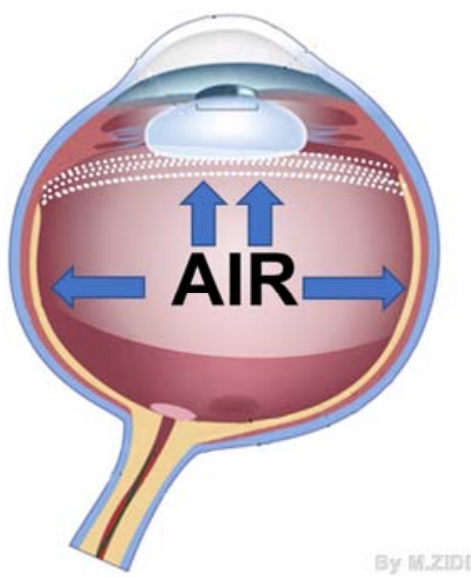

Figure 5. Complete air exchange-PFCL without pockets and slipping. 


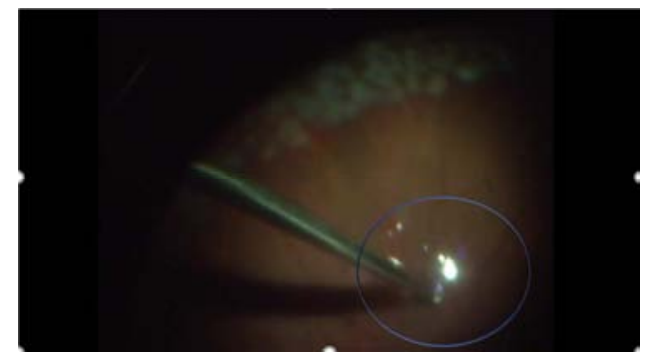

Figure 6. Patient H: Simulation of an incomplete exchange with formation of a pocket of liquid under air marked by the blue circle. This pocket was sufficient to cause a slip of the edge of the GRT.

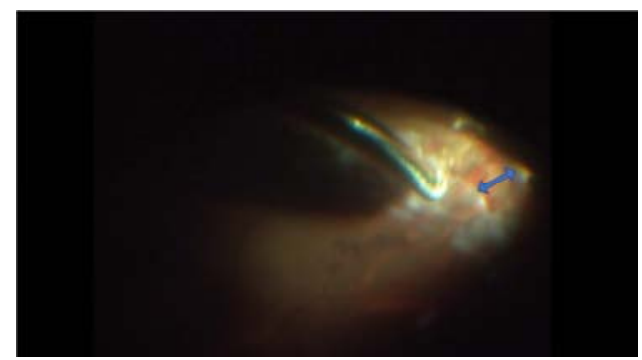

Figure 7. Patient H: The observed slip represented by the blue arrow. A simple eye to the ground positioning was sufficient in the case of this patient to treat this slip. If the slipping was important, a resumption of exchange would be necessary.

According to some authors, the visualization of the PFCL meniscus at the end of the exchange can be difficult under the air bubble, hence the risk of leaving small bubbles of PFCL, but another return to aspirate PFCL allows very often to remove it completely (Figures 8,9). The passage of PFCL bubbles by the tear under the retina is a complication described in the literature $[16,17]$. Often favored by a handling of PFCL in the presence of an undissected VR P leaving the retina retracted. In our series, we did not notice this complication.

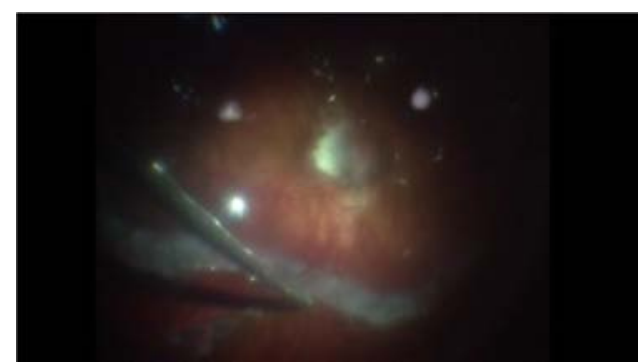

Figure 8. Patient $R: R D$ in the severely short-sighted: PFCL-air exchange with no slippage and retina perfectly reapplied.

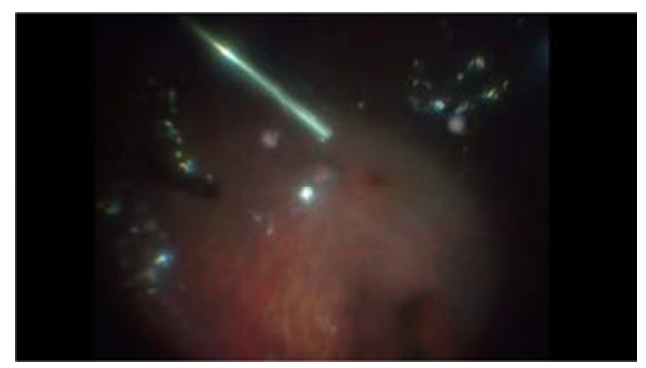

Figure 9. Patient $Z: R D$ in the case of the severely myopic patient: $P F C L$ AIR exchange with no fluid pocket under the retina and no slipping and a perfect retina reapplied.
The risk of retinal slipping is linked to an imbalance of forces between the re-application force of PFCL which becomes insufficient to resist the anteroposterior air pressure aggravated by the persistence of liquid under the retina and insufficient aspiration of the liquid interface between the air and PFCL [16]. This step of the PFCL-Air exchange is crucial. It success is based on the aspiration and drying of the serum regarding the edge of the GRT before removing the PFCL bubble on the posterior retina. Sometimes a complement of peripheral vitrectomy under fluid or air (dry vitrectomy) is necessary to have a correct tamponade contact as well with the tear and to remove the equivalent of a sponge which can retain serum and which will release it after removal of the PFCL. And this liquid may pass under the retina during the exchange and be responsible for the retinal slippage phenomenon. According to our theory, it's this passage of fluid during the exchange that will be the cause of the retinal slippage and not the contact of the retina with air in case of GRT. The diagrams below show how a retinal slippage can be observed. This slippage of the retina. will be proportional to the volume of the under retinal liquid. (Figures 6, 7 and 9).

The silicone-air exchange consists in injecting silicone with a pump in the vitreous cavity under air under visual control blindly. The silicone is injected at the bottom then the air rises up to the sclerotomies. The silicone is indeed heavier than the air and ensures a tamponade of the posterior pole towards the retinal periphery [18]. In the case of air-gas exchange, the principle of air-gas exchange is to replace all of the air filling the vitreous cavity by a non-expansive mixture of air and gas ensuring a more prolonged tamponade.

The $16 \%$ C2F6 mixture lasts 2 to 3 weeks, whereas the $12 \%$ C3F8 mixture lasts on average between 3 and 4 weeks. The choice of the gas is controversial and depends mostly on the surgeons' habits. According to some studies, there will be no significant difference between long term gas tamponade (C3F8) and silicone. In other series, the gas tamponade would be statistically correlated with the recurrence of Retinal detachment by giant tear [19, 20].

In our experience, we opt for a gas tamponade if the lower horn of the GRT is located above the meridians of $4 \mathrm{~h}$ and $8 \mathrm{~h}$ and if there is no VRP C. This has limited the use of gas in our series $(75 \%$ of the patients had a PVR C). Silicone oil tamponade is the most widely used given the location of the GRT and the presence of the PVR. The retinal slippage can be responsible for a badly post-operative retinopexy by shifting the edge of the tear treated, of postoperative folds and recurrence of RD.

In order to make easier the understanding of the retinal slippage, we have the analogy between its intraoperative occurrence in the course of PFCL-air exchange and a simulation by mathematical calculation (Figures 6, 7, 9, 10). Both confirm that the edge slipping of the DGR is proportional to the amount of liquid formed under the retinal at the end of the gas exchange (often in this case, the gas exchange is incomplete and not dry). If the exchange is fast or incomplete, the liquid will slide under the retina and form a bulging pocket under air. The roof of this back pocket (in 
green on the diagram) (Figure 10) is extensible and will be superior to that of the corresponding ocular wall which is inextensible (in yellow). The formed pocket will expand and lengthen its retinal side. And if we measure the length of this formed pocket and compare it to that of the corresponding choroid wall, we find out there is a difference in length which is none other than the length of the slipped retina. If the pocket is big, the slip will be too. It is believed that laser impacts made on the edge of the DGR helps to keep this edge in place if there is no liquid pocket formed. The slip will be visible intraoperatively by visualizing the laser impacts on the choroid. It can therefore be concluded that the edge of the DGR will tend to slip or shorten according to the volume of fluid formed under the retina after the exchange. The same volume will determine the surface area of the formed retinal pocket, which in turn determines the length of the slipped retina. Hence the conclusion: No under retinal liquid pocket formed at the end of the air exchange, no retinal slippage.

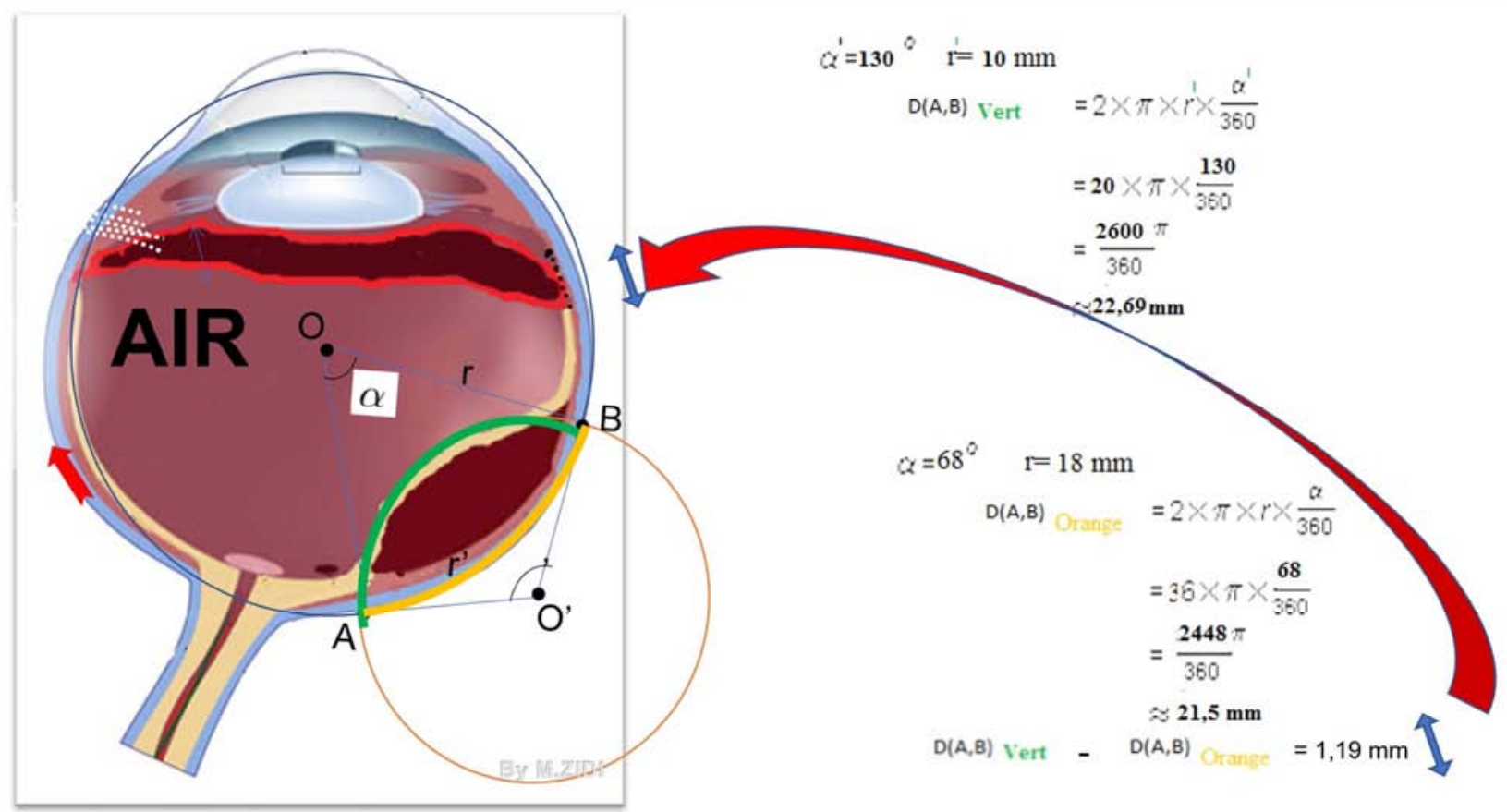

Figure 10. Diagrammatic illustration of an incomplete exchange with a liquid passage under the retina and formation of under retinal pocket. The calculation of the length of this pocket (in green) compared to that of the posterior choroidal wall (in yellow) shows a difference of 1.19 mm. This difference corresponds perfectly to the length of the slipping of the tear edge.

\section{Conclusion}

In $\mathrm{RD}$ by GRT, the phenomenon of retinal slippage constitutes a serious but preventable intraoperative complication. Knowledge and mastery of the technique of exchanging fluids and gases can prevent retinal slippage and ensure retinal reapplication without complications. This success is based solely on the lack of formation of subretinal fluid pockets by the PFCL-air exchange as has been shown by mathematical analysis. No under retinal liquid pocket formed at the end of the air exchange, no retinal slippage phenomenon. The gas ca be used as a safe alternative to silicone tamponade in higher localization GRT.

\section{References}

[1] Ang GS, Townend J, Lois N. Epidemiology of giant retinal tears in the United Kingdom: the British Giant Retinal Tear Epidemiology Eye Study (BGEES). Invest Ophthalmol Vis Sci 2010; 51 (9): 4781-4787.

[2] Lee SY, Ong SG, Wong DKW, Ang CL. Giant retinal tear management: an Asian experience. Eye 2009; 23: 601-605.

[3] Portella E, Moreira CA. Perfluorocarbonos líquidos In: Abujamra S, Ávila M, Barsante C, Farah ME, Gonçalves JO, et al. Retina e vítreo - clínica ecirurgia. São Paulo: Roca; 2000. p. $877-80$.

[4] Goezinne F., La Heij E. C., Berendschot T. T. et al. Low redetachment rate due to encircling scleral buckle in giant retinal tears treated with vitrectomy and silicone oil. Retina, 2008; 28: 485-92.

[5] Ambresin A, Wolfensberger TJ, Bovey EH. Management of giant retinal tears with vitrectomy, internal tamponade, and peripheral 360 degrees retinal photocoagulation. Retina. 2003; 23 (5): $622-8$

[6] Lavin M. Heavy liquids for postoperative tamponade. $\mathrm{Br} \mathrm{J}$ Ophthalmol. 1992; 76 (9): 513-4. Comment on: $\mathrm{Br} \mathrm{J}$ Ophthalmol. 1992; 76 (9): 525-8.

[7] Riemann C. D., Miller D. M., Foster R. E., Petersen M. R. Outcomes of transconjonctival suturless 25 -gauge vitrectomy with siliconeoil infusion. Retina, 2008; 28: 723-8.

[8] Shah C. P., Ho A. C., Regilio C. D. et al. Short-term outcomes of 25-gauge vitrectomy with silicone oil for repair of complicated retinal detachment. Retina, 2008; 28: 723-8. 
[9] Kumar V, Kumawat D, Bhari A, Chandra P. Twenty-fivegauge pars plana vitrectomy in complex retinal detachments associated with giant retinal tear. Retina 2018; 38: 670-677.

[10] Abrams GW, Garcia-Valenzuela E, Nanda SK. Retinotomies and Retinectomies. In: SCHACHAT AP, ed. Ryan's Retina. Vol Three. Sixth. Amsterdam, Netherlands: Elsevier; 2018.

[11] Hoerauf H., Laqua H. Severe emulsification after use of partially fluorinated alkane and silicone oil. Graefes Arch Clin Exp Ophthalmol, 2002; 2405: 131-6.

[12] Hoerauf H., Faude F., Menz D. H. et al. Determination of the solubility of perfluorocarbon liquids in silicone oil in vitro and in vivo. Retina, 2002; 22: 163-8.

[13] V. G. Madanagopalan Sandwich Technique with Anterior Silicone Oil and Posterior Perfluorocarbon Liquid for Intraoperative Retinal Stabilization in Eyes with Large Retinal Breaks J Ophthalmic Vis Res 2019; 14 (2): 232-235.

[14] Rouberol F., Feldman A., Denis P. et al. Étude prospective de 34 décollements de rétine par déchirure géante. J Fr Ophtalmol, 2010; 33: 23-30.
[15] Wong D., Williams R. L., German M. J. Exchange of perfluorodecalin for gas or oil: a model for avoiding slippage. Graefes Arch Clin Exp Ophthalmol, 1998; 236: 234-7.

[16] Berrod J.-P., Rozot P., Raspiller A., Thiery D. Fluid air exchange in vitreo retinal surgery. Int Ophthalmol, 1994; 18: 237-41.

[17] Kapetanios A. D., Donati G., Pournaras C. J. Déchirures géantes idiopathiques: traitement par vitrectomie et tamponnement temporaire par huile de silicone. $\mathrm{J}$ Fr Ophtalmol, 2000; 23: 1001-5.

[18] Yam J. C., Liu D. T., Lee V. Y. et al. Giant retinal tear after pneumatic retinopexy. Acta Ophthalmol, 2008; 86: 232-3.

[19] O., Ohji M. Intraocular gas tamponades. Seminar Oph, 2000; 15: 3-14.

[20] Parver LM, Lincoff H. Mechanics of intraocular gas. Invest Ophthalmol Vis Sci 1978; 17: 77-79. 